\title{
Gauge transformation and spectral decomposition for the Ishimori-II equations
}

\author{
Dmitry Pelinovsky ${ }^{1}$ and Derchyi $\mathbf{W u}^{2}$ \\ ${ }^{1}$ Department of Mathematics, McMaster University, Hamilton, Ontario, Canada L8S 4K1 \\ ${ }^{2}$ Institute of Mathematics, Academia Sinica, Taipei, Taiwan, Republic of China
}

Received 25 February 2003

Published 7 May 2003

Online at stacks.iop.org/JPhysA/36/5557

\begin{abstract}
We study the gauge transformation between spectral problems and their adjoints for the Ishimori-II (IS-II) and Davey-Stewartson-II (DS-II) equations. The commutativity between the gauge and adjoint transformations is proved. The commutativity is used for spectral decomposition and surface representation theorems for the Ishimori-II equations.
\end{abstract}

PACS numbers: $02.30 .1 \mathrm{k}, 02.30 . \mathrm{Tb}$

\section{Introduction}

Spectral decomposition is a fundamental result in spectral theory of self-adjoint operators. Many nonlinear partial differential equations in the inverse scattering transform method are associated with non-self-adjoint linear operators [AC91]. Since the regular direct and inverse scattering transforms are mutually reversible, non-self-adjoint linear operators must possess complete sets of eigenfunctions which are orthogonal with respect to eigenfunctions of the adjoint operators. If initial data evolve into algebraically decaying lump solutions of nonlinear equations, the spectral decomposition involves both the continuous and discrete spectrum of linear operators. Potentials of linear operators associated with lump solutions are defined in a weaker function space, compared to that required for a regular inverse scattering transform. Nevertheless, spectral decomposition theorems for time-dependent Schrödinger and Dirac operators with lump potentials were proved in [PSO0a, PSO0b] by methods of complex analysis based on the Riemann-Hilbert and $\bar{\partial}$ problems. It was shown that lumps of the KadomtsevPetviashvili-I (KP-I) equation are structurally stable in the spectral decomposition [PSO0a], while lumps of the Davey-Stewartson-II (DS-II) equations are structurally unstable and decay into continuous spectrum under generic perturbations of the initial data [GK96, PS00b].

In this paper, we study a complete set of eigenfunctions associated with the Ishimori-II (IS-II) equations,

$$
\begin{aligned}
& \mathbf{S}_{t}+\frac{1}{2} \mathbf{S} \times\left(\mathbf{S}_{x x}-\mathbf{S}_{y y}\right)+\phi_{x} \mathbf{S}_{y}+\phi_{y} \mathbf{S}_{x}=0 \\
& \phi_{x x}+\phi_{y y}-\mathbf{S} \cdot\left(\mathbf{S}_{x} \times \mathbf{S}_{y}\right)=0
\end{aligned}
$$


where $\mathbf{S}=\left(S_{1}, S_{2}, S_{3}\right)^{\mathrm{T}}=\mathbf{S}(x, y, t)$ is a three-component unit vector on the plane $(x, y)$ such that

$$
\mathbf{S} \cdot \mathbf{S}=1 \quad \lim _{|x|,|y| \rightarrow \infty} \mathbf{S}(x, y, t)=(0,0,1)^{\mathrm{T}}
$$

while $\phi(x, y, t)$ is scalar potential function generated by the topological charge density $\mathbf{S} \cdot\left(\mathbf{S}_{x} \times \mathbf{S}_{y}\right)$.

The IS-II equations describe time evolution of the system of static spin vortices on the plane $(x, y)$ [I84]. Static spin vortices are classified by the integer values of the topological charge:

$$
Q=\frac{1}{4 \pi} \iint_{\mathbb{R}^{2}} \mathbf{S} \cdot\left(\mathbf{S}_{x} \times \mathbf{S}_{y}\right) \mathrm{d} x \mathrm{~d} y .
$$

From mathematical point of view, the IS-II equations are remarkable because of their gauge equivalence to the DS-II equations [BC88, LS89]:

$$
\begin{aligned}
& \mathrm{i} q_{t}+\frac{1}{2}\left(q_{x x}-q_{y y}\right)+4\left(\varphi-|q|^{2}\right) q=0 \\
& \varphi_{x x}+\varphi_{y y}-2\left(|q|^{2}\right)_{x x}=0 .
\end{aligned}
$$

Linear non-self-adjoint operators for the IS-II equations (1.1) have potentials multiplied by $x$-derivative operators, compared to linear operators for the DS-II equations (1.3). As a result, a proof of the spectral decomposition theorem is particularly difficult for the linear operators of the IS-II equations. We prove the orthogonality and completeness of eigenfunctions by using the inverse scattering transform for both IS-II and DS-II equations, combined with the gauge transformation between these equations. Our approach is different from the straightforward computations used in the preceding papers [PS00a, PS00b]. The key property in our analysis is a commutativity (theorem 5.3) between the gauge and adjoint transformations of linear operators. The commutativity theorem deeply intertwines with the scattering theory for the IS-II and DS-II equations.

The paper is organized as follows. In section 2, we review the scattering data of the IS-II equations. In section 3, we study the gauge transformation between the IS-II and DS-II equations. An adjoint problem for the IS-II equations is introduced in section 4. In section 5, we study the gauge transformation between the adjoint problems and prove commutativity of the gauge and adjoint transformations. Section 6 presents applications to the spectral decomposition and surface representation theorems.

\section{Inverse scattering transform for the Ishimori-II equations}

The IS-II equations are the compatibility conditions of the over-determined linear system,

$$
\begin{aligned}
& \left(\mathrm{i} \partial_{y}+P \partial_{x}\right) \Psi=0 \\
& \left(2 \partial_{t}-2 \mathrm{i} P \partial_{x}^{2}-\left(\mathrm{i} P_{x}-P_{y} P-\mathrm{i} P \phi_{x}-\phi_{y}\right) \partial_{x}\right) \Psi=0
\end{aligned}
$$

where $\Psi=\left(\Psi_{1}, \Psi_{2}\right)^{\mathrm{T}}=\Psi(x, y, t), P=S_{1} \sigma_{1}+S_{2} \sigma_{2}+S_{3} \sigma_{3}=P(x, y, t)$ and $\sigma_{1}, \sigma_{2}, \sigma_{3}$ are Pauli matrices,

$$
\sigma_{1}=\left(\begin{array}{cc}
0 & 1 \\
1 & 0
\end{array}\right) \quad \sigma_{2}=\left(\begin{array}{cc}
0 & -\mathrm{i} \\
\mathrm{i} & 0
\end{array}\right) \quad \sigma_{3}=\left(\begin{array}{cc}
1 & 0 \\
0 & -1
\end{array}\right)
$$

such that

$$
P=\left(\begin{array}{cc}
S_{3} & S_{1}-\mathrm{i} S_{2} \\
S_{1}+\mathrm{i} S_{2} & -S_{3}
\end{array}\right)
$$


We introduce complex variables for elliptic problems on the plane $(x, y)$ :

$$
z=\frac{1}{2}(y-\mathrm{i} x) \quad \bar{z}=\frac{1}{2}(y+\mathrm{i} x)
$$

such that the spectral problem (2.1) can be rewritten for $\Psi=\Psi(z)$ :

$$
\left(\begin{array}{cc}
\partial_{\bar{z}} & 0 \\
0 & \partial_{z}
\end{array}\right) \Psi=\frac{1}{2}\left(P(z)-\sigma_{3}\right)\left(\partial_{z}-\partial_{\bar{z}}\right) \Psi
$$

on $z \in \mathbb{C}$. Here and henceforth we suppress the dependence of $\boldsymbol{\Psi}$ from $\bar{z}$ and $t$.

The $\bar{\partial}$-method was used in [BC88, K93] to solve the inverse scattering transform for the linear spectral problem (2.4). We introduce a fundamental matrix of solutions of (2.4),

$$
\Psi=\left(\boldsymbol{\mu} \mathrm{e}^{\mathrm{i} k z}, \chi \mathrm{e}^{-\mathrm{i} k \bar{z}}\right)
$$

where the vectors $\boldsymbol{\mu}=\left(\mu_{1}, \mu_{2}\right)^{\mathrm{T}}=\boldsymbol{\mu}(z, k)$ and $\chi=\left(\chi_{1}, \chi_{2}\right)^{\mathrm{T}}=\chi(z, k)$ solve the problems,

$$
\begin{aligned}
& \left(\begin{array}{cc}
\partial_{\bar{z}} & 0 \\
0 & \partial_{z}
\end{array}\right) \boldsymbol{\mu}+\mathrm{i} k\left(\begin{array}{cc}
0 & 0 \\
0 & 1
\end{array}\right) \boldsymbol{\mu}=\mathcal{P}_{k} \boldsymbol{\mu} \\
& \left(\begin{array}{cc}
\partial_{\bar{z}} & 0 \\
0 & \partial_{z}
\end{array}\right) \chi-\mathrm{i} k\left(\begin{array}{cc}
1 & 0 \\
0 & 0
\end{array}\right) \chi=\mathcal{P}_{k} \chi
\end{aligned}
$$

where

$$
\mathcal{P}_{k}=\frac{1}{2}\left(P(z)-\sigma_{3}\right)\left(\partial_{z}-\partial_{\bar{z}}+\mathrm{i} k\right) .
$$

The eigenvectors $\mu(z, k)$ and $\chi(z, k)$ satisfy the boundary conditions on the plane $(x, y)$ :

$$
\lim _{|z| \rightarrow \infty} \boldsymbol{\mu}(z, k)=\mathbf{e}_{1}=\left(\begin{array}{l}
1 \\
0
\end{array}\right) \quad \lim _{|z| \rightarrow \infty} \chi(z, k)=\mathbf{e}_{2}=\left(\begin{array}{l}
0 \\
1
\end{array}\right) .
$$

If $\mathbf{S}(z)$ is real-valued, the two fundamental solutions $\mu(z, k)$ and $\chi(z, k)$ of the problems (2.6) and (2.7) are related by means of the involution transformation:

$$
\chi(z, \bar{k})=\sigma \overline{\mu(z, k)} \quad \sigma=\left(\begin{array}{cc}
0 & -1 \\
1 & 0
\end{array}\right) .
$$

In the limit $|k| \rightarrow 0$, the eigenvector $\boldsymbol{\mu}(z, k)$ becomes a constant vector in $z$, which is uniquely defined by the normalization condition (2.9) as

$$
\lim _{|k| \rightarrow 0} \boldsymbol{\mu}(z, k)=\mathbf{e}_{1} .
$$

In the limit $|k| \rightarrow \infty$, the eigenvector $\boldsymbol{\mu}(z, k)$ becomes a vector $\boldsymbol{\mu}_{\infty}(z)$, which solves the algebraic problem,

$$
\left(\begin{array}{ll}
0 & 0 \\
0 & 1
\end{array}\right) \boldsymbol{\mu}_{\infty}(z)=\frac{1}{2}\left(P(z)-\sigma_{3}\right) \boldsymbol{\mu}_{\infty}(z)
$$

Components of the vector $\mu_{\infty}(z)$ are related by means of the stereographical variable,

$$
\frac{\mu_{2 \infty}}{\mu_{1 \infty}}=\frac{1-S_{3}}{S_{1}-\mathrm{i} S_{2}}=\frac{S_{1}+\mathrm{i} S_{2}}{1+S_{3}} .
$$

The stereographical variable in (2.13) satisfies the same family of nonlinear problem as the DS-II equations (1.3) [S92]. We define the matrix $m_{\infty}=m_{\infty}(z)$ as

$$
m_{\infty}(z)=\left(\boldsymbol{\mu}_{\infty}(z), \sigma \overline{\mu_{\infty}(z)}\right)=\left(\begin{array}{cc}
\mu_{1 \infty} & -\overline{\mu_{2 \infty}} \\
\mu_{2 \infty} & \overline{\mu_{1 \infty}}
\end{array}\right)
$$

such that $\operatorname{det}\left(m_{\infty}\right)=\left|\mu_{1 \infty}\right|^{2}+\left|\mu_{2 \infty}\right|^{2}$. As a result,

$$
P(z)=m_{\infty}(z) \sigma_{3} m_{\infty}^{-1}(z)
$$


or explicitly,

$$
S_{1}-\mathrm{i} S_{2}=\frac{2 \mu_{1 \infty} \overline{\mu_{2 \infty}}}{\left|\mu_{1 \infty}\right|^{2}+\left|\mu_{2 \infty}\right|^{2}} \quad S_{3}=\frac{\left|\mu_{1 \infty}\right|^{2}-\left|\mu_{2 \infty}\right|^{2}}{\left|\mu_{1 \infty}\right|^{2}+\left|\mu_{2 \infty}\right|^{2}} .
$$

The differential problem (2.6) can be transformed to the linear integral equation by means of the Green function,

$$
\begin{aligned}
& \mu_{1}(z, k)=1+\frac{1}{2 \pi \mathrm{i}} \iint \frac{\mathrm{d} z^{\prime} \wedge \mathrm{d} \bar{z}^{\prime}}{z^{\prime}-z}\left(\mathcal{P}_{k} \boldsymbol{\mu}\right)_{1}\left(z^{\prime}\right) \\
& \mu_{2}(z, k)=\frac{1}{2 \pi \mathrm{i}} \iint \frac{\mathrm{d} z^{\prime} \wedge \mathrm{d} \bar{z}^{\prime}}{\bar{z}^{\prime}-\bar{z}}\left(\mathcal{P}_{k} \boldsymbol{\mu}\right)_{2}\left(z^{\prime}\right) \mathrm{e}^{\mathrm{i} k\left(z^{\prime}-z\right)+\mathrm{i} \bar{k}\left(\bar{z}^{\prime}-\bar{z}\right)} .
\end{aligned}
$$

The same Fredholm integral equations (2.17) occur in the spectral problem for the DS-II equations [PS00b]. The integral equations (2.17) have a unique solution if the potential function $P(z)$ in $L^{2}\left(\mathbb{R}^{2}\right) \cap L^{1}\left(\mathbb{R}^{2}\right)$ [BC84]. If the potential function $P(z)$ in $L^{2}\left(\mathbb{R}^{2}\right)$ but not in $L^{1}\left(\mathbb{R}^{2}\right)$, the integral equations (2.17) may have homogeneous solutions, corresponding to bound states [FA84, APP89]. Bound states $\boldsymbol{\Phi}_{j}(z)$, are decaying solutions of the equation,

$$
\left(\begin{array}{cc}
\partial_{\bar{z}} & 0 \\
0 & \partial_{z}
\end{array}\right) \boldsymbol{\Phi}_{j}+\mathrm{i} k_{j}\left(\begin{array}{ll}
0 & 0 \\
0 & 1
\end{array}\right) \boldsymbol{\Phi}_{j}=\mathcal{P}_{k_{j}} \boldsymbol{\Phi}_{j}
$$

subject to the boundary conditions,

$$
\lim _{|z| \rightarrow \infty} z \Phi_{j}(z)=\mathbf{e}_{1} .
$$

Each eigenvalue $k_{j}$ is double, since there exists a linearly independent eigenfunction $\Phi_{j}^{\prime}(z)$ solving the same equation (2.18) and satisfying the boundary conditions

$$
\lim _{|z| \rightarrow \infty} z \Phi_{j}^{\prime}(z) \mathrm{e}^{\mathrm{i} k_{j} z+\mathrm{i} \overline{k_{j} z}}=\mathbf{e}_{2} .
$$

The two linearly independent eigenfunctions are related as

$$
\boldsymbol{\Phi}_{j}^{\prime}(z)=\sigma \overline{\boldsymbol{\Phi}_{j}(z)} \mathrm{e}^{-\mathrm{i}\left(k_{j} z+\overline{k_{j} z}\right)} .
$$

We assume that there is a finite number (say $n$ ) of eigenvalues $k_{j}$ on the plane $k \in \mathbb{C}$, such that $k_{j} \neq 0, j=1, \ldots, n$. It follows from (2.17) that the eigenfunction $\mu(z, k)$ satisfies the $\bar{\partial}$ problem in the domain $\mathcal{D}=\left\{k \in \mathbb{C}: k \neq k_{j}, j=1, \ldots, n\right\}$, such that

$$
\frac{\partial \boldsymbol{\mu}}{\partial \bar{k}}=b(k) \mathbf{N}_{\mu}(z, k)
$$

where the spectral coefficient $b(k)$ is defined as

$$
b(k)=\frac{1}{2 \pi} \iint \mathrm{d} z \wedge \mathrm{d} \bar{z}\left(\mathcal{P}_{k} \boldsymbol{\mu}\right)_{2}(z) \mathrm{e}^{\mathrm{i}(k z+\overline{k z})}
$$

and the linearly independent eigenfunction $\mathbf{N}_{\mu}(z, k)$ solves the same integral equation (2.17) but with a different (oscillatory) behaviour at infinity:

$$
\begin{aligned}
& N_{\mu 1}(z, k)=\frac{1}{2 \pi \mathrm{i}} \iint \frac{\mathrm{d} z^{\prime} \wedge \mathrm{d} \bar{z}^{\prime}}{z^{\prime}-z}\left(\mathcal{P}_{k} \mathbf{N}_{\mu}\right)_{1}\left(z^{\prime}\right) \\
& N_{\mu 2}(z, k)=\mathrm{e}^{-\mathrm{i}(k z+\overline{k z})}+\frac{1}{2 \pi \mathrm{i}} \iint \frac{\mathrm{d} z^{\prime} \wedge \mathrm{d} \bar{z}^{\prime} \mathrm{e}^{\mathrm{i}\left(k\left(z^{\prime}-z\right)+\overline{\left.k\left(z^{\prime}-z\right)\right)}\right.}}{\bar{z}^{\prime}-\bar{z}}\left(\mathcal{P}_{k} \mathbf{N}_{\mu}\right)_{2}\left(z^{\prime}\right) .
\end{aligned}
$$

For real-valued $\mathbf{S}(z)$, there exists a local relation between two linearly independent eigenfunctions $\boldsymbol{\mu}(z, k)$ and $\mathbf{N}_{\mu}(z, k)$ :

$$
\mathbf{N}_{\mu}(z, k)=\sigma \overline{\boldsymbol{\mu}(z, k)} \mathrm{e}^{-\mathrm{i}(k z+\overline{k z})} .
$$


When the eigenfunction $\boldsymbol{\mu}(z, k)$ approaches to the points $k=k_{j}, j=1, \ldots, n$, where the bound state exists, it has a singular (pole) behaviour in the limit $k \rightarrow k_{j}$. We assume the following limiting behaviour of $\mu(z, k)$ :

$$
\lim _{k \rightarrow k_{j}}\left(\boldsymbol{\mu}(z, k)-\frac{\mathrm{i}}{k-k_{j}} \boldsymbol{\Phi}_{j}(z)\right)=\left(z+z_{j}\right) \boldsymbol{\Phi}_{j}(z)+c_{j} \boldsymbol{\Phi}_{j}^{\prime}(z)
$$

where $z_{j}, c_{j}$ are arbitrary constants. We notice that this limiting behaviour introduces a constraint on the class of potentials as for the DS-II equations in [PSO0b]. The constraint is always satisfied for lump potentials. No such constraints were introduced in the original paper [APP89], where a different limiting behaviour of $\boldsymbol{\mu}(z, k)$ was suggested as $k \rightarrow k_{j}$ :

$$
\lim _{k \rightarrow k_{j}} \frac{\partial}{\partial k}\left(\left(k-k_{j}\right) \boldsymbol{\mu}(z, k)\right)=\left(z+z_{j}\right) \Phi_{j}(z)+c_{j} \boldsymbol{\Phi}_{j}^{\prime}(z) .
$$

We neglect this discrepancy between (2.26) and (2.27), since the spectral decomposition and structural instability of points $k=k_{j}$ does not depend on the types of singular behaviour of $\boldsymbol{\mu}(z, k)$ as $k \rightarrow k_{j}$.

Solving the $\bar{\partial}$ problem (2.22) on plane $k \in \mathbb{C}$ subject to the boundary conditions $\lim _{|k| \rightarrow \infty} \boldsymbol{\mu}(z, k)=\boldsymbol{\mu}_{\infty}(z)$ and using the limiting conditions (2.26) at the poles $k=k_{j}, j=$ $1, \ldots, n$, we derive the expansion formula,

$\boldsymbol{\mu}(z, k)=\boldsymbol{\mu}_{\infty}(z)+\sum_{j=1}^{n} \frac{\mathrm{i}}{k-k_{j}} \boldsymbol{\Phi}_{j}(z)+\frac{1}{2 \pi \mathrm{i}} \iint \frac{\mathrm{d} k^{\prime} \wedge \mathrm{d} \bar{k}^{\prime}}{k^{\prime}-k} b\left(k^{\prime}\right) \mathbf{N}_{\mu}\left(z, k^{\prime}\right)$

Using the limiting condition (2.11) as $k \rightarrow 0$, we express the limiting function $\mu_{\infty}(z)$ explicitly as

$$
\boldsymbol{\mu}_{\infty}(z)=\mathbf{e}_{1}+\sum_{j=1}^{n} \frac{\mathrm{i}}{k_{j}} \boldsymbol{\Phi}_{j}(z)-\frac{1}{2 \pi \mathrm{i}} \iint \frac{\mathrm{d} k^{\prime} \wedge \mathrm{d} \bar{k}^{\prime}}{k^{\prime}} b\left(k^{\prime}\right) \mathbf{N}_{\mu}\left(z, k^{\prime}\right) .
$$

As a result, we find an explicit expansion formula for $\boldsymbol{\mu}(z, k)$ as

$\boldsymbol{\mu}(z, k)=\mathbf{e}_{1}+\sum_{j=1}^{n} \frac{\mathrm{i} k}{k_{j}\left(k-k_{j}\right)} \boldsymbol{\Phi}_{j}(z)+\frac{k}{2 \pi \mathrm{i}} \iint \frac{\mathrm{d} k^{\prime} \wedge \mathrm{d} \bar{k}^{\prime}}{k^{\prime}\left(k^{\prime}-k\right)} b\left(k^{\prime}\right) \mathbf{N}_{\mu}\left(z, k^{\prime}\right)$.

Using the limiting relations as $k \rightarrow k_{j}$, we close the system (2.30) with a set of algebraic equations for $j=1, \ldots, n$ :

$$
\begin{gathered}
\left(z+z_{j}-\frac{\mathrm{i}}{k_{j}}\right) \boldsymbol{\Phi}_{j}(z)+c_{j} \boldsymbol{\Phi}_{j}^{\prime}(z)=\mathbf{e}_{1}+\sum_{l \neq j}^{n} \frac{\mathrm{i} k_{j}}{k_{l}\left(k_{j}-k_{l}\right)} \boldsymbol{\Phi}_{l}(z) \\
+\frac{k_{j}}{2 \pi \mathrm{i}} \iint \frac{\mathrm{d} k^{\prime} \wedge \mathrm{d} \bar{k}^{\prime}}{k^{\prime}\left(k^{\prime}-k_{j}\right)} b\left(k^{\prime}\right) \mathbf{N}_{\mu}\left(z, k^{\prime}\right) .
\end{gathered}
$$

We conclude this section with an explicit example of a single lump solution of the IS-II equations. Let $b(k)=0$ and $n=1$ in the equations above. Then the algebraic system (2.31) is decoupled from (2.30) and it has a unique solution:

$$
\boldsymbol{\Phi}_{1 j}(z)=\frac{\bar{z}+\bar{z}_{j}+\frac{\mathrm{i}}{k_{j}}}{\left|z+z_{j}-\frac{\mathrm{i}}{k_{j}}\right|^{2}+\left|c_{j}\right|^{2}} \quad \boldsymbol{\Phi}_{2 j}(z)=\frac{-c_{j} \mathrm{e}^{-\mathrm{i}\left(k_{j} z+\overline{k_{j} z}\right)}}{\left|z+z_{j}-\frac{\mathrm{i}}{k_{j}}\right|^{2}+\left|c_{j}\right|^{2}} .
$$

The eigenfunction $\boldsymbol{\mu}(z, k)$ has a pole decomposition representation,

$$
\boldsymbol{\mu}(z, k)=\mathbf{e}_{1}+\frac{\mathrm{i} k}{k_{j}\left(k-k_{j}\right)} \boldsymbol{\Phi}_{j}(z) \quad \boldsymbol{\mu}_{\infty}(z)=\mathbf{e}_{1}+\frac{\mathrm{i}}{k_{j}} \boldsymbol{\Phi}_{j}(z) .
$$


The single lump solution for the vector $\mathbf{S}(z)$ takes the explicit form:

$$
\begin{aligned}
& S_{1}-\mathrm{i} S_{2}=\frac{2 \mathrm{i} \bar{c}_{j}\left[\left(z+z_{j}\right)\left(\bar{z}+\bar{z}_{j}+\frac{\mathrm{i}}{k_{j}}\right)+\left|c_{j}\right|^{2}\right] \mathrm{e}^{\mathrm{i}\left(k_{j} z+\overline{k_{j} z}\right)}}{\bar{k}_{j}\left(\left|z+z_{j}\right|^{2}+\left|c_{j}\right|^{2}\right)\left(\left|z+z_{j}-\frac{\mathrm{i}}{k_{j}}\right|^{2}+\left|c_{j}\right|^{2}\right)} \\
& S_{3}=1-\frac{2\left|c_{j}\right|^{2}}{\left|k_{j}\right|^{2}\left(\left|z+z_{j}\right|^{2}+\left|c_{j}\right|^{2}\right)\left(\left|z+z_{j}-\frac{\mathrm{i}}{k_{j}}\right|^{2}+\left|c_{j}\right|^{2}\right)} .
\end{aligned}
$$

We note that the single lump solution of the IS-II equations is different from the single vortex solution found in [I84] with the Hirota's method. However, the single vortex solution in [I84] can be recovered from the single lump solution (2.32)-(2.35) in the singular limit $k_{j} \rightarrow 0$, with the appropriate shifts of $z_{j}$ and $\bar{z}_{j}$. In our paper, we will be working only with the lump solutions which are derived in the inverse scattering transform for a general non-singular case $k_{j} \neq 0, j=1, \ldots, n$.

\section{Gauge transformation between the DS-II and IS-II equations}

The DS-II equations (1.3) in complex coordinates (2.3) are related with the following linear operator:

$$
\left(\begin{array}{cc}
\partial_{\bar{z}} & 0 \\
0 & \partial_{z}
\end{array}\right) \boldsymbol{\Psi}_{\mathrm{DS}}=Q(z) \boldsymbol{\Psi}_{\mathrm{DS}}
$$

where

$$
Q(z)=\left(\begin{array}{cc}
0 & -q(z) \\
\bar{q}(z) & 0
\end{array}\right)
$$

The fundamental matrix of the linear operator for the DS-II equations is $\boldsymbol{\Psi}_{\mathrm{DS}}=$ $\left(\mu_{\mathrm{DS}} \mathrm{e}^{\mathrm{i} k z}, \chi_{\mathrm{DS}} \mathrm{e}^{-\mathrm{i} k \bar{z}}\right)$, where $\chi_{\mathrm{DS}}(z, \bar{k})=\sigma \overline{\mu_{\mathrm{DS}}(z, k)}$ and $\mu_{\mathrm{DS}}(z, k)$ satisfies the problem:

$$
\left(\begin{array}{cc}
\partial_{\bar{z}} & 0 \\
0 & \partial_{z}
\end{array}\right) \mu_{\mathrm{DS}}+\mathrm{i} k\left(\begin{array}{cc}
0 & 0 \\
0 & 1
\end{array}\right) \mu_{\mathrm{DS}}=Q(z) \mu_{\mathrm{DS}} .
$$

Linear spectral problems (2.4) and (3.1) for the IS-II and DS-II equations are related to the gauge transformation reported in [BC88, LS89].

Lemma 3.1. Let $m_{\infty}(z)$ be defined by (2.14) such that $P(z)=m_{\infty} \sigma_{3} m_{\infty}^{-1}$. Solutions of the linear system (2.4) are equivalent to solutions of the linear system (3.1) with the gauge transformation

$$
\Psi_{\mathrm{DS}}(z, k)=m_{\infty}^{-1}(z) \Psi(z, k)
$$

such that

$$
Q(z)=\left(\mathcal{D}_{z} m_{\infty}^{-1}\right) m_{\infty} \quad \mathcal{D}_{z}=\left(\begin{array}{cc}
\partial_{\bar{z}} & 0 \\
0 & \partial_{z}
\end{array}\right)
$$

Proof. We first show that if $\mathbf{m}=(\boldsymbol{\mu}(z, k), \chi(z, k))$ with $\boldsymbol{\mu}, \chi$ satisfying (2.6), (2.7) and $\mathbf{m}_{\mathrm{DS}}=m_{\infty}^{-1} \mathbf{m}=\left(\boldsymbol{\mu}_{\mathrm{DS}}, \chi_{\mathrm{DS}}\right)$, then $\boldsymbol{\mu}_{\mathrm{DS}}$ solves the problem (3.3), where the matrix $Q(z)$ has zero diagonal entries, while $\chi_{\mathrm{DS}}(z, \bar{k})=\sigma \overline{\mu_{\mathrm{DS}}(z, k)}$. For simplicity, we consider only the case $n=0$. We rewrite $(2.28)$ in the operator form,

$$
\mathbf{m}=m_{\infty}+\mathcal{C} \mathcal{T}(\mathbf{m})
$$


where $\mathcal{C}$ is the Cauchy integral operator,

$$
\mathcal{C}(\mathbf{f})=\frac{1}{2 \pi \mathrm{i}} \iint \frac{\mathrm{d} k^{\prime} \wedge \mathrm{d} \bar{k}^{\prime}}{k^{\prime}-k} \mathbf{f}\left(k^{\prime}\right)
$$

and $\mathcal{T}$ is the right multiplicative operator, such that

$$
\mathcal{T}(\boldsymbol{f})=\mathcal{T}\left(\begin{array}{ll}
f_{11} & f_{12} \\
f_{21} & f_{22}
\end{array}\right)(z, k)=\boldsymbol{f}(z, \bar{k}) \mathbf{S}_{z}(k) .
$$

Here the scattering matrix $\mathbf{S}_{z}(k)$ is

$$
\mathbf{S}_{z}(k)=\left(\begin{array}{cc}
0 & \mathrm{e}^{\mathrm{i}(\bar{k} z+k \bar{z})} \overline{b(\bar{k})} \\
\mathrm{e}^{-\mathrm{i}(k z+\overline{k z})} b(k) & 0
\end{array}\right)
$$

and we have used (2.10) and (2.25). Multiplying (3.6) with $m_{\infty}^{-1}$, we find that

$$
m_{\infty}^{-1} \mathbf{m}=I+\mathcal{C} m_{\infty}^{-1} \mathcal{T}(\mathbf{m})=I+\mathcal{C} \mathcal{T}\left(m_{\infty}^{-1} \mathbf{m}\right)
$$

where $I$ is the 2-by-2 identity matrix. We apply the operator,

$$
\mathcal{D}_{k} \boldsymbol{f}=\mathcal{D}_{z} \boldsymbol{f}+k(\operatorname{ad} \mathcal{J} \boldsymbol{f})=\left(\begin{array}{cc}
\partial_{\bar{z}} & 0 \\
0 & \partial_{z}
\end{array}\right) \boldsymbol{f}-\frac{\mathrm{i}}{2} k\left[\sigma_{3}, \boldsymbol{f}\right]
$$

to both sides of (3.10) and use the commutativity property,

$$
\left[\mathcal{D}_{k}, \mathcal{T}\right] \boldsymbol{f}=0 .
$$

As a result, we obtain

$$
\mathcal{D}_{k}\left(m_{\infty}^{-1} \mathbf{m}\right)=\mathcal{D}_{k} \mathcal{C} \mathcal{T}\left(m_{\infty}^{-1} \mathbf{m}\right)=\mathcal{C} \mathcal{T} \mathcal{D}_{k}\left(m_{\infty}^{-1} \mathbf{m}\right)+Q(z)
$$

where

$$
\begin{aligned}
& Q(z)=\left[\mathcal{D}_{k}, \mathcal{C}\right] \mathcal{T}\left(m_{\infty}^{-1} \mathbf{m}\right)=-\frac{1}{2 \pi \mathrm{i}} \operatorname{ad} \mathcal{J} \iint \mathrm{d} k \wedge \mathrm{d} \bar{k} \mathcal{T}\left(m_{\infty}^{-1} \mathbf{m}\right)(z, k) \\
&=\frac{1}{2 \pi} \iint \mathrm{d} k \wedge \mathrm{d} \bar{k}\left(\begin{array}{cc}
0 & \left(\mathcal{T}\left(m_{\infty}^{-1} \mathbf{m}\right)\right)_{12}(z, k) \\
\left(\mathcal{T}\left(m_{\infty}^{-1} \mathbf{m}\right)\right)_{21}(z, \bar{k}) & 0
\end{array}\right) .
\end{aligned}
$$

We use the relation (3.10) and the commutativity relation,

$$
[Q(z), \mathcal{T}] \mathbf{f}=0
$$

and finish the first part of the proof,

$$
\mathcal{D}_{k}\left(m_{\infty}^{-1} \mathbf{m}\right)=\left(I-\mathcal{C} \mathcal{D}_{k}\right)^{-1} Q(z)=Q(z)\left(I-\mathcal{C} \mathcal{D}_{k}\right)^{-1} I=Q(z) m_{\infty}^{-1} \mathbf{m}
$$

In the second part of the proof, we show that if $\Psi$ solves (2.4) with $P(z)=m_{\infty} \sigma_{3} m_{\infty}^{-1}$ and $\Psi=m_{\infty} \boldsymbol{\Psi}_{\mathrm{DS}}$, then $\boldsymbol{\Psi}_{\mathrm{DS}}$ solves (3.1), where $Q(z)$ is related to $m_{\infty}$ by means of (3.5). We rewrite (2.4) in the form

$$
\mathcal{D}_{z}\left(m_{\infty} \boldsymbol{\Psi}_{\mathrm{DS}}\right)=\frac{1}{2}\left(m_{\infty} \sigma_{3} m_{\infty}^{-1}-\sigma_{3}\right)\left(\partial_{z}-\partial_{\bar{z}}\right) m_{\infty} \boldsymbol{\Psi}_{\mathrm{DS}}
$$

Straightforward differentiations transform (3.16) to the form

$$
\begin{aligned}
& \left(\begin{array}{cc}
\partial_{\bar{z}} \mu_{1 \infty} & -\partial_{\bar{z}} \overline{\mu_{2 \infty}} \\
\partial_{z} \mu_{2 \infty} & \partial_{z} \overline{\mu_{1 \infty}}
\end{array}\right) \boldsymbol{\Psi}_{\mathrm{DS}}+m_{\infty} \mathcal{D}_{z} \boldsymbol{\Psi}_{\mathrm{DS}} \\
& \quad=\frac{1}{\operatorname{det}\left(m_{\infty}\right)}\left(\begin{array}{cc}
-\left|\mu_{2 \infty}\right|^{2} & \mu_{1 \infty} \overline{\mu_{2 \infty}} \\
\overline{\mu_{1 \infty}} \mu_{2 \infty} & \left|\mu_{2 \infty}\right|^{2}
\end{array}\right)\left(\left(\partial_{z}-\partial_{\bar{z}}\right) m_{\infty}\right) \boldsymbol{\Psi}_{\mathrm{DS}}
\end{aligned}
$$

which further transforms to the form

$$
m_{\infty} \mathcal{D}_{z} \Psi_{\mathrm{DS}}=m_{\infty} Q(z) \Psi_{\mathrm{DS}}
$$


where

$Q(z)=\frac{1}{\operatorname{det}\left(m_{\infty}\right)}\left(\begin{array}{cc}-\overline{\mu_{1 \infty}} \partial_{\bar{z}} \mu_{1 \infty}-\overline{\mu_{2 \infty}} \partial_{\bar{z}} \mu_{2 \infty} & \overline{\mu_{1 \infty}} \partial_{\bar{z}} \overline{\mu_{2 \infty}}-\overline{\mu_{2 \infty}} \partial_{\bar{z}} \overline{\mu_{1 \infty}} \\ \mu_{2 \infty} \partial_{z} \mu_{1 \infty}-\mu_{1 \infty} \partial_{z} \mu_{2 \infty} & -\mu_{2 \infty} \partial_{z} \overline{\mu_{2 \infty}}-\mu_{1 \infty} \partial_{z} \overline{\mu_{1 \infty}}\end{array}\right)$.

Explicit differentiations in (3.5) shows that representations (3.5) and (3.19) are the same.

Corollary 3.2. Components of the transformation matrix $m_{\infty}(z)$ in (2.14) satisfy the constraints:

$$
\begin{aligned}
& q(z)=\frac{1}{\operatorname{det}\left(m_{\infty}\right)}\left(\overline{\mu_{2 \infty}} \partial_{\bar{z}} \overline{\mu_{1 \infty}}-\overline{\mu_{1 \infty}} \partial_{\bar{z}} \overline{\mu_{2 \infty}}\right) \\
& 0=\mu_{1 \infty} \partial_{z} \overline{\mu_{1 \infty}}+\mu_{2 \infty} \partial_{z} \overline{\mu_{2 \infty}} .
\end{aligned}
$$

We continue the explicit example (2.32)-(2.35) and compute the gauge transformation between the single lump solutions of the IS-II and DS-II equations. It follows from (2.33) that

$\mu_{1 \infty}=1+\frac{\mathrm{i}\left(\bar{z}+\bar{z}_{j}+\frac{\mathrm{i}}{k_{j}}\right)}{k_{j}\left(\left|z+z_{j}-\frac{\mathrm{i}}{k_{j}}\right|^{2}+\left|c_{j}\right|^{2}\right)} \quad \mu_{2 \infty}=\frac{-\mathrm{i} c_{j} \mathrm{e}^{-\mathrm{i}\left(k_{j} z+\overline{k_{j} z}\right)}}{k_{j}\left(\left|z+z_{j}-\frac{\mathrm{i}}{k_{j}}\right|^{2}+\left|c_{j}\right|^{2}\right)}$

such that

$$
\operatorname{det}\left(m_{\infty}\right)=\frac{\left|z+z_{j}\right|^{2}+\left|c_{j}\right|^{2}}{\left|z+z_{j}-\frac{\mathrm{i}}{k_{j}}\right|^{2}+\left|c_{j}\right|^{2}}
$$

Constraints (3.20) and (3.21) are satisfied with

$$
q=\frac{\bar{c}_{j} \mathrm{e}^{\mathrm{i}\left(k_{j} z+\overline{k_{j} z}\right)}}{\left|z+z_{j}\right|^{2}+\left|c_{j}\right|^{2}} .
$$

Therefore, the gauge transformation (3.5) generates a single lump solution of the DS-II equations (1.3) from a single lump solution of the IS-II equations (1.1). The eigenfunctions of the Lax operator for the DS-II equations are generated by the gauge transformation $\boldsymbol{\mu}_{\mathrm{DS}}=m_{\infty}^{-1} \boldsymbol{\mu}$ to the form

$$
\boldsymbol{\mu}_{\mathrm{DS}}(z, k)=\mathbf{e}_{1}+\frac{\mathrm{i}}{k-k_{j}} \boldsymbol{\Phi}_{\mathrm{DS}}(z)
$$

and

$$
\Phi_{\mathrm{DS} 1}(z)=\frac{\bar{z}+\bar{z}_{j}}{\left|z+z_{j}\right|^{2}+\left|c_{j}\right|^{2}} \quad \Phi_{\mathrm{DS} 2}(z)=\frac{-c_{j} \mathrm{e}^{-\mathrm{i}\left(k_{j} z+\overline{k_{j} z}\right)}}{\left|z+z_{j}\right|^{2}+\left|c_{j}\right|^{2}}
$$

\section{The adjoint linear problem}

Let $\mathcal{L}$ be a linear operator that maps $L^{2}\left(\mathbb{R}^{2}\right)$ to $L^{2}\left(\mathbb{R}^{2}\right)$. We define the adjoint operator $\mathcal{L}^{a}$ by the inner product for two vector functions $f, g \in L^{2}\left(\mathbb{R}^{2}\right)$ :

$$
\langle\mathbf{f} \mid \mathcal{L} \mathbf{g}\rangle=\left\langle\mathcal{L}^{+} \mathbf{f} \mid \mathbf{g}\right\rangle \quad \mathcal{L}^{+}=\overline{\mathcal{L}^{a}}
$$

where the standard inner product is

$$
\langle\mathbf{f} \mid \mathbf{g}\rangle=\iint\left(\bar{f}_{1} g_{1}+\bar{f}_{2} g_{2}\right) \mathrm{d} z \wedge \mathrm{d} \bar{z}
$$

If $\mathcal{L} \Psi=\mathbf{0}$ is a linear problem with an eigenfunction $\boldsymbol{\Psi} \in L^{2}\left(\mathbb{R}^{2}\right)$, then $\mathcal{L}^{a} \boldsymbol{\Psi}^{a}=\mathbf{0}$ is the adjoint problem with an adjoint eigenfunction $\Psi^{a} \in L^{2}\left(\mathbb{R}^{2}\right)$, which takes the form:

$$
\left(\begin{array}{cc}
\partial_{z} & 0 \\
0 & \partial_{\bar{z}}
\end{array}\right) \Psi^{a}=-\frac{1}{2}\left(\partial_{z}-\partial_{\bar{z}}\right)\left(P^{+}(z)-\sigma_{3}\right) \Psi^{a}
$$


where $P^{+}(z)=P(z)$. The fundamental matrix of adjoint eigenfunctions for the IS-II equations is

$$
\Psi^{a}(z)=\left(\chi^{a}(z, k) \mathrm{e}^{\mathrm{i} k \bar{z}}, \boldsymbol{\mu}^{a}(z, k) \mathrm{e}^{-\mathrm{i} k z}\right)
$$

where $\chi^{a}(z, k)$ and $\boldsymbol{\mu}^{a}(z, k)$ solve the adjoint problems to (2.6) and (2.7), respectively:

$$
\begin{aligned}
& \left(\begin{array}{cc}
\partial_{z} & 0 \\
0 & \partial_{\bar{z}}
\end{array}\right) \chi^{a}+\mathrm{i} k\left(\begin{array}{ll}
0 & 0 \\
0 & 1
\end{array}\right) \chi^{a}=-\mathcal{P}_{k}^{a} \chi^{a} \\
& \left(\begin{array}{cc}
\partial_{z} & 0 \\
0 & \partial_{\bar{z}}
\end{array}\right) \boldsymbol{\mu}^{a}-\mathrm{i} k\left(\begin{array}{cc}
1 & 0 \\
0 & 0
\end{array}\right) \boldsymbol{\mu}^{a}=-\mathcal{P}_{k}^{a} \boldsymbol{\mu}^{a}
\end{aligned}
$$

where

$$
\mathcal{P}_{k}^{a}=\frac{1}{2}\left(\partial_{z}-\partial_{\bar{z}}-\mathrm{i} k\right)\left(P^{+}(z)-\sigma_{3}\right) .
$$

The eigenvectors $\chi^{a}(z, k)$ and $\boldsymbol{\mu}^{a}(z, k)$ satisfy the boundary conditions on the plane $(x, y)$ :

$$
\lim _{|z| \rightarrow \infty} \chi^{a}(z, k)=\mathbf{e}_{1} \quad \lim _{|z| \rightarrow \infty} \boldsymbol{\mu}^{a}(z, k)=\mathbf{e}_{2} .
$$

If $\mathbf{S}(z)$ is real-valued, the two fundamental solutions $\chi^{a}(z, k)$ and $\boldsymbol{\mu}^{a}(z, k)$ of the problems (4.5) and (4.6) are related by means of the involution transformation:

$$
\chi^{a}(z, \bar{k})=-\sigma \overline{\mu^{a}(z, k)}
$$

In the limit $|k| \rightarrow \infty$, the eigenvector $\boldsymbol{\mu}^{a}(z, k)$ becomes a vector $\boldsymbol{\mu}_{\infty}^{a}(z)$, which solves the algebraic problem,

$$
\left(\begin{array}{ll}
1 & 0 \\
0 & 0
\end{array}\right) \boldsymbol{\mu}_{\infty}^{a}(z)=-\frac{1}{2}\left(P(z)-\sigma_{3}\right) \mu_{\infty}^{a}(z)
$$

Components of the vector $\boldsymbol{\mu}_{\infty}^{a}(z)$ are related by means of the adjoint stereographical variable,

$$
\frac{\mu_{2 \infty}^{a}}{\mu_{1 \infty}^{a}}=-\frac{1+S_{3}}{S_{1}-\mathrm{i} S_{2}}=-\frac{S_{1}+\mathrm{i} S_{2}}{1-S_{3}} .
$$

We define the adjoint matrix $m_{\infty}^{a}=m_{\infty}^{a}(z)$ as

$$
m_{\infty}^{a}(z)=\left(-\sigma \overline{\boldsymbol{\mu}_{\infty}^{a}(z)}, \boldsymbol{\mu}_{\infty}^{a}(z)\right)=\left(\begin{array}{cc}
\overline{\mu_{2 \infty}^{a}} & \mu_{1 \infty}^{a} \\
-\overline{\mu_{1 \infty}^{a}} & \mu_{2 \infty}^{a}
\end{array}\right)
$$

such that $\operatorname{det}\left(m_{\infty}^{a}\right)=\left|\mu_{1 \infty}^{a}\right|^{2}+\left|\mu_{2 \infty}^{a}\right|^{2}$. As a result,

$$
P^{+}(z)=m_{\infty}^{a}(z) \sigma_{3}\left(m_{\infty}^{a}\right)^{-1}(z)
$$

or explicitly,

$$
S_{1}-\mathrm{i} S_{2}=\frac{-2 \mu_{1 \infty}^{a} \overline{\mu_{2 \infty}^{a}}}{\left|\mu_{1 \infty}^{a}\right|^{2}+\left|\mu_{2 \infty}^{a}\right|^{2}} \quad S_{3}=\frac{\left|\mu_{2 \infty}^{a}\right|^{2}-\left|\mu_{1 \infty}^{a}\right|^{2}}{\left|\mu_{1 \infty}^{a}\right|^{2}+\left|\mu_{2 \infty}^{a}\right|^{2}} .
$$

The differential problem (4.6) can be transformed to the linear integral equation by means of the Green function,

$$
\begin{aligned}
& \mu_{1}^{a}(z, k)=-\frac{1}{2 \pi \mathrm{i}} \iint \frac{\mathrm{d} z^{\prime} \wedge \mathrm{d} \bar{z}^{\prime}}{\bar{z}^{\prime}-\bar{z}}\left(\mathcal{P}_{k}^{a} \mu^{a}\right)_{1}\left(z^{\prime}\right) \mathrm{e}^{-\mathrm{i} k\left(z^{\prime}-z\right)-\mathrm{i} \bar{k}\left(\bar{z}^{\prime}-\bar{z}\right)} \\
& \mu_{2}^{a}(z, k)=1-\frac{1}{2 \pi \mathrm{i}} \iint \frac{\mathrm{d} z^{\prime} \wedge \mathrm{d} \bar{z}^{\prime}}{z^{\prime}-z}\left(\mathcal{P}_{k}^{a} \mu^{a}\right)_{2}\left(z^{\prime}\right) .
\end{aligned}
$$


If the potential function $P(z)$ in $L^{2}\left(\mathbb{R}^{2}\right)$ but not in $L^{1}\left(\mathbb{R}^{2}\right)$, the integral equations (4.15) may have homogeneous solutions, corresponding to bound states. The bound states $\boldsymbol{\Phi}_{j}^{a}(z)$ are decaying solutions of the equation,

$$
\left(\begin{array}{cc}
\partial_{z} & 0 \\
0 & \partial_{\bar{z}}
\end{array}\right) \boldsymbol{\Phi}_{j}^{a}-\mathrm{i} k_{j}^{a}\left(\begin{array}{cc}
1 & 0 \\
0 & 0
\end{array}\right) \boldsymbol{\Phi}_{j}^{a}=-\mathcal{P}_{k_{j}^{a}}^{a} \boldsymbol{\Phi}_{j}^{a}
$$

subject to the boundary conditions,

$$
\lim _{|z| \rightarrow \infty} z \Phi_{j}^{a}(z)=\mathbf{e}_{2}
$$

Each eigenvalue $k_{j}^{a}$ of the adjoint problem is double, since there exists a linearly independent eigenfunction $\Phi_{j}^{a \prime}(z)$ solving the same equation (4.16) and related to $\Phi_{j}^{a}(z)$ by means of the involution:

$$
\boldsymbol{\Phi}_{j}^{a \prime}(z)=-\sigma \overline{\Phi_{j}^{a}(z)} \mathrm{e}^{\mathrm{i}\left(k_{j}^{a} z+\overline{k_{j}^{a}} z\right)}
$$

It follows from (4.15) that the eigenfunction $\mu^{a}(z, k)$ satisfies the $\bar{\partial}$ problem in the domain $\mathcal{D}^{a}=\left\{k \in \mathbb{C}: k \neq k_{j}^{a}, j=1, \ldots, n\right\}$, such that

$$
\frac{\partial \boldsymbol{\mu}^{a}}{\partial \bar{k}}=-b^{a}(k) \mathbf{N}_{\mu}^{a}(z, k)
$$

where the spectral coefficient $b^{a}(k)$ is defined as

$$
b^{a}(k)=\frac{1}{2 \pi} \iint \mathrm{d} z \wedge \mathrm{d} \bar{z}\left(\mathcal{P}_{k}^{a} \boldsymbol{\mu}^{a}\right)_{1}(z) \mathrm{e}^{-\mathrm{i}(k z+\overline{k z})}
$$

and the linearly independent eigenfunction $\mathbf{N}_{\mu}^{a}(z, k)$ solves the same integral equation (4.15) but with a different (oscillatory) behaviour at infinity,

$$
\begin{aligned}
& N_{\mu 1}^{a}(z, k)=\mathrm{e}^{\mathrm{i}(k z+\overline{k z})}-\frac{1}{2 \pi \mathrm{i}} \iint \frac{\mathrm{d} z^{\prime} \wedge \mathrm{d} \bar{z}^{\prime}}{\bar{z}^{\prime}-\bar{z}}\left(\mathcal{P}_{k}^{a} \mathbf{N}_{\mu}^{a}\right)_{1}\left(z^{\prime}\right) \mathrm{e}^{-\mathrm{i}\left(k\left(z^{\prime}-z\right)+\overline{k\left(z^{\prime}-z\right)}\right)} \\
& N_{\mu 2}^{a}(z, k)=-\frac{1}{2 \pi \mathrm{i}} \iint \frac{\mathrm{d} z^{\prime} \wedge \mathrm{d} \bar{z}^{\prime}}{z^{\prime}-z}\left(\mathcal{P}_{k}^{a} \mathbf{N}_{\mu}^{a}\right)_{2}\left(z^{\prime}\right)
\end{aligned}
$$

For real-valued $\mathbf{S}(z)$, there exists a local relation between two linearly independent eigenfunctions $\boldsymbol{\mu}^{a}(z, k)$ and $\mathbf{N}_{\mu}^{a}(z, k)$ :

$$
\mathbf{N}_{\mu}^{a}(z, k)=-\sigma \overline{\boldsymbol{\mu}^{a}(z, k)} \mathrm{e}^{\mathrm{i}(k z+\overline{k z})} .
$$

When the eigenfunction $\boldsymbol{\mu}(z, k)$ approaches to the points $k=k_{j}^{a}, j=1, \ldots, n$, where the bound states exist, it has a singular (pole) behaviour in the limit $k \rightarrow k_{j}^{a}$. We assume the following limiting behaviour of $\boldsymbol{\mu}^{a}(z, k)$ :

$$
\lim _{k \rightarrow k_{j}^{a}}\left(\boldsymbol{\mu}^{a}(z, k)+\frac{\mathrm{i}}{k-k_{j}^{a}} \boldsymbol{\Phi}_{j}^{a}(z)\right)=\left(z+z_{j}^{a}\right) \boldsymbol{\Phi}_{j}^{a}(z)+c_{j}^{a} \boldsymbol{\Phi}_{j}^{a \prime}(z)
$$

where $z_{j}^{a}, c_{j}^{a}$ are arbitrary constants.

Solving the $\bar{\partial}$ problem (4.19) on plane $k \in \mathbb{C}$ subject to the boundary conditions $\lim _{|k| \rightarrow \infty} \boldsymbol{\mu}^{a}(z, k)=\boldsymbol{\mu}_{\infty}^{a}(z)$ and using the limiting conditions (4.23) at the poles $k=k_{j}^{a}, j=$ $1, \ldots, n$, we derive the expansion formula,

$\boldsymbol{\mu}^{a}(z, k)=\boldsymbol{\mu}_{\infty}^{a}(z)-\sum_{j=1}^{n} \frac{\mathrm{i}}{k-k_{j}^{a}} \boldsymbol{\Phi}_{j}^{a}(z)-\frac{1}{2 \pi \mathrm{i}} \iint \frac{\mathrm{d} k^{\prime} \wedge \mathrm{d} \bar{k}^{\prime}}{k^{\prime}-k} b^{a}\left(k^{\prime}\right) \mathbf{N}_{\mu}^{a}\left(z, k^{\prime}\right)$. 
With the use of the limiting relations as $k \rightarrow k_{j}^{a}$, we close the system (4.24) with a set of algebraic equations for $j=1, \ldots, n$ :

$$
\begin{gathered}
\left(z+z_{j}^{a}\right) \boldsymbol{\Phi}_{j}^{a}(z)+c_{j}^{a} \boldsymbol{\Phi}_{j}^{a \prime}(z)=\boldsymbol{\mu}_{\infty}^{a}(z)-\sum_{l \neq j}^{n} \frac{\mathrm{i}}{k_{j}^{a}-k_{l}^{a}} \boldsymbol{\Phi}_{l}^{a}(z) \\
-\frac{1}{2 \pi \mathrm{i}} \iint \frac{\mathrm{d} k^{\prime} \wedge \mathrm{d} \bar{k}^{\prime}}{k^{\prime}-k_{j}^{a}} b^{a}\left(k^{\prime}\right) \mathbf{N}_{\mu}^{a}\left(z, k^{\prime}\right) .
\end{gathered}
$$

The system of equations (4.24) and (4.25) is incomplete, since $\boldsymbol{\mu}_{\infty}^{a}(z)$ is unknown. The adjoint problem (4.6) does not exhibit any simple solution at $k=0$, in contrast to the direct problem (2.6). We complete the system (4.24)-(4.25) by deriving the relation between $m_{\infty}^{a}$ and $m_{\infty}$. The relation appears as the commutativity condition between the gauge and adjoint transformations.

\section{Commutativity between the gauge and adjoint transformations}

The adjoint problem for the DS-II equations is

$$
\left(\begin{array}{cc}
\partial_{z} & 0 \\
0 & \partial_{\bar{z}}
\end{array}\right) \Psi_{\mathrm{DS}}^{a}=-Q^{+}(z) \Psi_{\mathrm{DS}}^{a}
$$

where $Q^{+}(z)=-Q(z)$. The fundamental matrix of the adjoint operator for the DS-II equations is $\Psi_{\mathrm{DS}}^{a}=\left(\chi_{\mathrm{DS}}^{a} \mathrm{e}^{\mathrm{i} k \bar{z}}, \mu_{\mathrm{DS}}^{a} \mathrm{e}^{-\mathrm{i} k z}\right)$, where $\chi_{\mathrm{DS}}^{a}(z, \bar{k})=-\sigma \overline{\mu_{\mathrm{DS}}^{a}(z, k)}$. Adjoint spectral problems (4.3) and (5.1) for the IS-II and DS-II equations are related with the adjoint gauge transformation.

Lemma 5.1. Let $m_{\infty}^{a}(z)$ be defined by (4.12) such that $P^{+}(z)=m_{\infty}^{a} \sigma_{3}\left(m_{\infty}^{a}\right)^{-1}$. Solutions of the linear system (4.3) are equivalent to solutions of the linear system (5.1) with the gauge transformation,

$$
\Psi_{\mathrm{DS}}^{a}(z, k)=\left(m_{\infty}^{a}\right)^{-1}(z) \Psi^{a}(z, k)
$$

such that

$$
Q^{+}(z)=\left(m_{\infty}^{a}\right)^{-1}\left(D_{\bar{z}}\left(m_{\infty}^{a}\right)^{\mathrm{T}}\right)^{\mathrm{T}} \quad \mathcal{D}_{\bar{z}}=\left(\begin{array}{cc}
\partial_{z} & 0 \\
0 & \partial_{\bar{z}}
\end{array}\right)
$$

Proof. We show by direct differentiation that if $\Psi^{a}$ satisfies (4.3) and $\Psi^{a}=m_{\infty}^{a} \Psi_{\mathrm{DS}}^{a}$, then $\Psi_{\mathrm{DS}}^{a}$ satisfies (5.1) with $Q^{+}(z)$ given in (5.3). Using (4.13), we notice that

$$
-\frac{1}{2}\left(P^{+}(z)-\sigma_{3}\right) m_{\infty}^{a}=\left(\begin{array}{cc}
0 & \mu_{1 \infty}^{a} \\
\mu_{1 \infty}^{a} & 0
\end{array}\right) .
$$

Substitution $\Psi^{a}=m_{\infty}^{a} \Psi_{\mathrm{DS}}^{a}$ reduces (4.3) to the form:

$$
\mathcal{D}_{\bar{z}} m_{\infty}^{a} \Psi_{\mathrm{DS}}^{a}=\left(\partial_{z}-\partial_{\bar{z}}\right)\left(\begin{array}{cc}
0 & \mu_{1 \infty}^{a} \\
\mu_{1 \infty}^{a} & 0
\end{array}\right) \Psi_{\mathrm{DS}}^{a} .
$$

Differentiation expands (5.5) to the form

$$
m_{\infty}^{a} \mathcal{D}_{\bar{z}} \Psi_{\mathrm{DS}}^{a}=-\left(\begin{array}{cc}
\partial_{z} \overline{\mu_{2 \infty}^{a}} & \partial_{\bar{z}} \mu_{1 \infty}^{a} \\
-\partial_{z} \overline{\mu_{1 \infty}^{a}} & \partial_{\bar{z}} \mu_{2 \infty}^{a}
\end{array}\right) \Psi_{\mathrm{DS}}^{a} .
$$

Inverting $m_{\infty}^{a}$ and matching with (5.1), we express $Q^{+}(z)$ in terms of $m_{\infty}^{a}$ as 


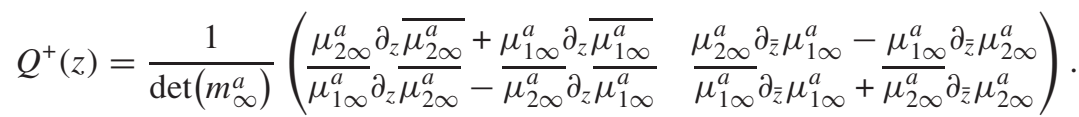

Direct differentiation shows equivalence of representations (5.3) and (5.7).

Corollary 5.2. Components of the transformation matrix $m_{\infty}^{a}(z)$ in (4.12) satisfy the constraints:

$$
\begin{aligned}
& q(z)=\frac{1}{\overline{\mu_{2 \infty}^{a}}} \partial_{\bar{z}} \mu_{1 \infty}^{a} \\
& 0=\mu_{1 \infty}^{a} \partial_{z} \overline{\mu_{1 \infty}^{a}}+\mu_{2 \infty}^{a} \partial_{z} \overline{\mu_{2 \infty}^{a}} .
\end{aligned}
$$

if $Q^{+}=-Q$.

We plot the diagram of the gauge and adjoint transformations for the IS-II and DS-II equations:

$$
\begin{array}{ccc}
\text { IS-II (2.4) for } P(z) & \rightarrow & \text { DS-II (3.1) for } Q(z) \\
\downarrow & \vdots \\
\text { adjoint IS-II (4.3) for } P^{+}(z) & \rightarrow & \text { adjoint DS-II (5.1) for } Q^{+}(z) .
\end{array}
$$

The gauge transformation from IS-II to DS-II is given by lemma 3.1. The adjoint transformation from IS-II to adjoint IS-II is described in section 4 for $P^{+}=P$. The gauge transformation from adjoint IS-II to adjoint DS-II is given by lemma 5.1. It remains to show that the system (5.1) is indeed the adjoint system of (3.1) with $Q^{+}=-Q$. This would serve as a commutativity condition between the gauge and adjoint transformations. We prove the commutativity condition and close the diagram (5.10) with an important relation between $m_{\infty}^{a}(z)$ and $m_{\infty}(z)$.

Theorem 5.3. The gauge transformation and the adjoint transformation are commutative if and only if

$$
m_{\infty}^{a}=\left(m_{\infty}^{-1}\right)^{+}=\frac{m_{\infty}}{\operatorname{det}\left(m_{\infty}\right)}
$$

such that

$$
\mu_{1 \infty}^{a}=-\frac{\overline{\mu_{2 \infty}}}{\left|\mu_{1 \infty}\right|^{2}+\left|\mu_{2 \infty}\right|^{2}} \quad \mu_{2 \infty}^{a}=\frac{\overline{\mu_{1 \infty}}}{\left|\mu_{1 \infty}\right|^{2}+\left|\mu_{2 \infty}\right|^{2}}
$$

and $\operatorname{det}\left(m_{\infty}^{a}\right) \operatorname{det}\left(m_{\infty}\right)=1$.

Proof. A relation between $m_{\infty}$ and $m_{\infty}^{a}$ follows from comparison of representations (2.15) and (4.13):

$$
P^{+}(z)=\left(m_{\infty}^{-1}\right)^{+} \sigma_{3} m_{\infty}^{+}=m_{\infty}^{a} \sigma_{3}\left(m_{\infty}^{a}\right)^{-1}
$$

Another relation follows from comparison of representations (3.5) and (5.3):

$$
Q^{+}=m_{\infty}^{+}\left(D_{\bar{z}}\left(m_{\infty}^{-1}\right)^{+\mathrm{T}}\right)^{\mathrm{T}}=\left(m_{\infty}^{a}\right)^{-1}\left(D_{\bar{z}}\left(m_{\infty}^{a}\right)^{\mathrm{T}}\right)^{\mathrm{T}}
$$

The constraint (5.11) is a sufficient condition for the relations (5.13) and (5.14). We prove that this is also a necessary condition for commutability of the gauge and adjoint transformations. By comparing (2.13) and (4.11), we derive a relation between $\boldsymbol{\mu}_{\infty}$ and $\boldsymbol{\mu}_{\infty}^{a}$ :

$$
\mu_{1 \infty} \overline{\mu_{1 \infty}^{a}}+\mu_{2 \infty} \overline{\mu_{2 \infty}^{a}}=0 .
$$


Using combinations of constraints (3.20), (3.21), (5.8) and (5.9), combined with the relation (5.15), we also derive that

$$
\mu_{1 \infty}^{a} \mu_{2 \infty}=\overline{\mu_{1 \infty}^{a}} \overline{\mu_{2 \infty}} \quad \mu_{2 \infty}^{a} \mu_{1 \infty}=\overline{\mu_{2 \infty}^{a}} \overline{\mu_{1 \infty}} .
$$

The relations (5.15) and (5.16) are satisfied if and only if

$$
\mu_{1 \infty}^{a}=-c(z) \overline{\mu_{2 \infty}} \quad \mu_{2 \infty}^{a}=c(z) \overline{\mu_{1 \infty}}
$$

where $c \in \mathbb{R}$. Using constraints (3.20), (5.8), combined with the relation (5.17), we find that

$$
c(z)=\frac{c_{0}}{\operatorname{det}\left(m_{\infty}\right)}
$$

where $c_{0}$ is constant. It follows from the asymptotic limits,

$$
\lim _{|z| \rightarrow \infty} m_{\infty}(z)=I \quad \lim _{|z| \rightarrow \infty} m_{\infty}^{a}(z)=I
$$

that $c_{0}=1$ and the formula (5.11) is thus proved.

We can develop an alternative proof of theorem 5.3, without using lemma 5.1 and corollary 5.2. The diagram of the gauge and adjoint transformations for the IS-II and DS-II equations can be closed as

$$
\begin{array}{ccc}
\text { IS-II (2.4) for } P(z) & \rightarrow & \text { DS-II (3.1) for } Q(z) \\
\downarrow & & \downarrow \\
\text { adjoint IS-II (4.3) for } P^{+}(z) & \cdots & \text { adjoint DS-II (5.1) for } Q^{+}(z) .
\end{array}
$$

Here we will use the adjoint system for DS-II equations (5.1) with $Q^{+}=-Q$ and show that the system is related to the adjoint system for IS-II equations (4.3) with $P^{+}=P$ by means of the gauge transformation:

$$
\Psi^{a}(z, k)=\frac{m_{\infty}(z)}{\operatorname{det}\left(m_{\infty}(z)\right)} \boldsymbol{\Psi}_{\mathrm{DS}}^{a}(z, k) .
$$

This relation is equivalent to the gauge transformation between the adjoint IS-II and DS-II equations in lemma 5.1, where $m_{\infty}^{a}(z)$ is given by (5.11).

The left-hand side of (4.3) is written explicitly in the form,

$$
\begin{aligned}
& D_{\bar{z}}\left(\frac{m_{\infty}}{\operatorname{det} m_{\infty}}\left(\begin{array}{c}
\Psi_{1, \mathrm{DS}}^{a} \\
\Psi_{2, \mathrm{DS}}^{a}
\end{array}\right)\right) \\
&=\frac{1}{\operatorname{det} m_{\infty}}\left(\begin{array}{c}
\left(\partial_{z} \mu_{1 \infty}\right) \Psi_{1, \mathrm{DS}}^{a}+\mu_{1 \infty} \partial_{z} \Psi_{1, \mathrm{DS}}^{a}-\left(\partial_{z} \bar{\mu}_{2 \infty}\right) \Psi_{2, \mathrm{DS}}^{a}-\bar{\mu}_{2 \infty} \partial_{z} \Psi_{2, \mathrm{DS}}^{a} \\
\left(\partial_{\bar{z}} \mu_{2 \infty}\right) \Psi_{1, \mathrm{DS}}^{a}+\mu_{2 \infty} \partial_{\bar{z}} \Psi_{1, \mathrm{DS}}^{a}+\left(\partial_{\bar{z}} \bar{\mu}_{1 \infty}\right) \Psi_{2, \mathrm{DS}}^{a}+\bar{\mu}_{1 \infty} \partial_{\bar{z}} \Psi_{2, \mathrm{DS}}^{a}
\end{array}\right) \\
&-\frac{1}{\left(\operatorname{det} m_{\infty}\right)^{2}}\left(\begin{array}{c}
\left(\mu_{1 \infty} \Psi_{1, \mathrm{DS}}^{a}-\bar{\mu}_{2 \infty} \Psi_{2, \mathrm{DS}}^{a}\right)\left[\left(\partial_{z} \mu_{1 \infty}\right) \bar{\mu}_{1 \infty}+\left(\partial_{z} \mu_{2 \infty}\right) \bar{\mu}_{2 \infty}\right] \\
\left(\mu_{2 \infty} \Psi_{1, \mathrm{DS}}^{a}+\bar{\mu}_{1 \infty} \Psi_{2, \mathrm{DS}}^{a}\right)\left[\mu_{1 \infty} \partial_{\bar{z}} \bar{\mu}_{1 \infty}+\mu_{2 \infty} \partial_{\bar{z}} \bar{\mu}_{2 \infty}\right]
\end{array}\right) .
\end{aligned}
$$

We apply the gauge transformation $Q=\mathcal{D}_{z}\left(m_{\infty}^{-1}\right) m_{\infty}$ to the adjoint DS-II system (5.1) and find that

$$
\begin{aligned}
D_{\bar{z}}\left(\frac{m_{\infty}}{\operatorname{det}\left(m_{\infty}\right)}\left(\begin{array}{c}
\Psi_{1, \mathrm{DS}}^{a} \\
\Psi_{2, \mathrm{DS}}^{a}
\end{array}\right)\right)=\frac{1}{\operatorname{det} m_{\infty}}\left(\begin{array}{c}
\left(\partial_{z} \mu_{1 \infty}\right) \Psi_{1, \mathrm{DS}}^{a}-\left(\partial_{z} \bar{\mu}_{2 \infty}\right) \Psi_{2, \mathrm{DS}}^{a}-\bar{\mu}_{2 \infty} \partial_{z} \Psi_{2, \mathrm{DS}}^{a} \\
\left(\partial_{\bar{z}} \mu_{2 \infty}\right) \Psi_{1, \mathrm{DS}}^{a}+\mu_{2 \infty}\left(\partial_{\bar{z}} \Psi_{1, \mathrm{DS}}^{a}\right)+\left(\partial_{\bar{z}} \bar{\mu}_{1 \infty}\right) \Psi_{2, \mathrm{DS}}^{a}
\end{array}\right) \\
+\frac{1}{\left(\operatorname{det} m_{\infty}\right)^{2}}\left(\begin{array}{c}
\mu_{1 \infty}\left(\bar{\mu}_{1 \infty} \partial_{\bar{z}} \bar{\mu}_{2 \infty}-\bar{\mu}_{1 \infty} \partial_{\bar{z}} \bar{\mu}_{2 \infty}\right) \Psi_{1, \mathrm{DS}}^{a} \\
\left(\bar{\mu}_{1 \infty} \mu_{1 \infty}-\mu_{1 \infty} \partial_{z} \mu_{2 \infty}\right) \Psi_{1, \mathrm{DS}}^{a}
\end{array}\right) \\
+\frac{1}{\left(\operatorname{det} m_{\infty}\right)^{2}}\left(\begin{array}{c}
-\left|\mu_{1 \infty}\right|^{2}\left(\partial_{z} \mu_{1 \infty}\right) \Psi_{1, \mathrm{DS}}^{a}+\bar{\mu}_{1 \infty} \bar{\mu}_{2 \infty}\left(\partial_{z} \mu_{1 \infty}\right) \Psi_{2, \mathrm{DS}}^{a} \\
-\left|\mu_{1 \infty}\right|^{2}\left(\partial_{\bar{z}} \bar{\mu}_{1 \infty}\right) \Psi_{2, \mathrm{DS}}^{a}-\mu_{2 \infty} \mu_{1 \infty}\left(\partial_{\bar{z}} \bar{\mu}_{1 \infty}\right) \Psi_{1, \mathrm{DS}}^{a}
\end{array}\right) \\
+\frac{1}{\left(\operatorname{det} m_{\infty}\right)^{2}}\left(\begin{array}{c}
-\mu_{1 \infty} \bar{\mu}_{2 \infty}\left(\partial_{z} \mu_{2 \infty}\right) \Psi_{1, \mathrm{DS}}^{a}+\bar{\mu}_{2 \infty}^{2}\left(\partial_{z} \mu_{2 \infty}\right) \Phi_{21}^{a} \\
-\mu_{2 \infty} \bar{\mu}_{1 \infty}\left(\partial_{\bar{z}} \bar{\mu}_{2 \infty}\right) \Psi_{2, \mathrm{DS}}^{a}-\mu_{2 \infty}^{2}\left(\partial_{\bar{z}} \bar{\mu}_{2 \infty}\right) \Psi_{1, \mathrm{DS}}^{a}
\end{array}\right) .
\end{aligned}
$$


On the other hand, the right-hand side of (4.3) can be rewritten as

$$
\begin{aligned}
& -\frac{1}{2}\left(\partial_{z}-\partial_{\bar{z}}\right)\left(P(z)-\sigma_{3}\right)\left(\frac{m_{\infty}}{\operatorname{det}\left(m_{\infty}\right)}\left(\begin{array}{c}
\Psi_{1, \mathrm{DS}}^{a} \\
\Psi_{2, \mathrm{DS}}^{a}
\end{array}\right)\right) \\
& =\frac{1}{\operatorname{det} m_{\infty}}\left(\begin{array}{l}
\left(\partial_{\bar{z}} \bar{\mu}_{2 \infty}\right) \Psi_{2, \mathrm{DS}}^{a}+\bar{\mu}_{2 \infty} \partial_{\bar{z}} \Psi_{2, \mathrm{DS}}^{a}-\left(\partial_{z} \bar{\mu}_{2 \infty}\right) \Psi_{2, \mathrm{DS}}^{a}-\bar{\mu}_{2 \infty} \partial_{z} \Psi_{2, \mathrm{DS}}^{a} \\
\left(\partial_{\bar{z}} \mu_{2 \infty}\right) \Psi_{1, \mathrm{DS}}^{a}+\mu_{2 \infty} \partial_{\bar{z}} \Psi_{1, \mathrm{DS}}^{a}-\left(\partial_{z} \mu_{2 \infty}\right) \Psi_{1, \mathrm{DS}}^{a}-\mu_{2 \infty} \partial_{z} \Psi_{1, \mathrm{DS}}^{a}
\end{array}\right) \\
& -\frac{1}{\left(\operatorname{det} m_{\infty}\right)^{2}}\left(\begin{array}{l}
\bar{\mu}_{2 \infty} \Psi_{2, \mathrm{DS}}^{a}\left(\mu_{1 \infty} \partial_{\bar{z}} \bar{\mu}_{1 \infty}+\mu_{2 \infty} \partial_{\bar{z}} \bar{\mu}_{2 \infty}-\left(\partial_{z} \mu_{1 \infty}\right) \bar{\mu}_{1 \infty}-\left(\partial_{z} \mu_{2 \infty}\right) \bar{\mu}_{2 \infty}\right) \\
\mu_{2 \infty} \Psi_{1, \mathrm{DS}}^{a}\left(\mu_{1 \infty} \partial_{\bar{z}} \bar{\mu}_{1 \infty}+\mu_{2 \infty} \partial_{\bar{z}} \bar{\mu}_{2 \infty}-\left(\partial_{z} \mu_{1 \infty}\right) \bar{\mu}_{1 \infty}-\left(\partial_{z} \mu_{2 \infty}\right) \bar{\mu}_{2 \infty}\right)
\end{array}\right) .
\end{aligned}
$$

We prove that the right-hand sides of (5.23) and (5.24) are equal by showing that the following two expressions are equal

$$
\begin{array}{r}
\frac{1}{\operatorname{det} m_{\infty}}\left(\begin{array}{c}
\left(\partial_{z} \mu_{1 \infty}\right) \Psi_{1, \mathrm{DS}}^{a}+\mu_{1 \infty} \frac{\left(\bar{\mu}_{1 \infty} \bar{\mu}_{2 \infty}-\bar{\mu}_{2 \infty} \partial_{\bar{z}} \bar{\mu}_{1 \infty}\right) \Psi_{2, \mathrm{DS}}^{a}}{\operatorname{det} m_{\infty}} \\
\left(\partial_{\bar{z}} \bar{\mu}_{1 \infty}\right) \Psi_{2, \mathrm{DS}}^{a}+\bar{\mu}_{1 \infty} \frac{\left(\mu_{2 \infty} \partial_{z} \mu_{1 \infty}-\mu_{1 \infty} \partial_{z} \mu_{2 \infty}\right) \Psi_{1, \mathrm{DS}}^{a}}{\operatorname{det} m_{\infty}}
\end{array}\right) \\
+\frac{1}{\left(\operatorname{det} m_{\infty}\right)^{2}}\left(\begin{array}{c}
-\left|\mu_{1 \infty}\right|^{2}\left(\partial_{z} \mu_{1 \infty}\right) \Psi_{1, \mathrm{DS}}^{a}-\mu_{1 \infty} \bar{\mu}_{2 \infty}\left(\partial_{z} \mu_{2 \infty}\right) \Psi_{1, \mathrm{DS}}^{a} \\
-\left|\mu_{1 \infty}\right|^{2}\left(\partial_{\bar{z}} \bar{\mu}_{1 \infty}\right) \Psi_{2, \mathrm{DS}}^{a}-\mu_{2 \infty} \bar{\mu}_{1 \infty}\left(\partial_{\bar{z}} \bar{\mu}_{2 \infty}\right) \Psi_{2, \mathrm{DS}}^{a}
\end{array}\right)
\end{array}
$$

and

$$
\begin{gathered}
\frac{1}{\operatorname{det} m_{\infty}}\left(\begin{array}{c}
\left(\partial_{\bar{z}} \bar{\mu}_{2 \infty}\right) \Psi_{2, \mathrm{DS}}^{a}+\bar{\mu}_{2 \infty} \frac{\left(\mu_{2 \infty} \partial_{z} \mu_{1 \infty}-\mu_{1 \infty} \partial_{z} \mu_{2 \infty}\right) \Psi_{1, \mathrm{DS}}^{a}}{\operatorname{det} m_{\infty}} \\
-\left(\partial_{z} \mu_{2 \infty}\right) \Psi_{1, \mathrm{DS}}^{a}-\mu_{2 \infty} \frac{\left(\bar{\mu}_{1 \infty} \partial_{\bar{z}} \bar{\mu}_{2 \infty}-\bar{\mu}_{2 \infty} \partial_{\bar{z}} \bar{\mu}_{1 \infty}\right) \Psi_{2, \mathrm{DS}}^{a}}{\operatorname{det} m_{\infty}}
\end{array}\right) \\
-\frac{1}{\left(\operatorname{det} m_{\infty}\right)^{2}}\left(\begin{array}{c}
\left|\mu_{2 \infty}\right|^{2}\left(\partial_{\bar{z}} \bar{\mu}_{2 \infty}\right) \Psi_{2, \mathrm{DS}}^{a}+\mu_{1 \infty} \bar{\mu}_{2 \infty}\left(\partial_{\bar{z}} \bar{\mu}_{1 \infty}\right) \Psi_{2, \mathrm{DS}}^{a} \\
-\left|\mu_{2 \infty}\right|^{2}\left(\partial_{z} \mu_{2 \infty}\right) \Psi_{1, \mathrm{DS}}^{a}-\bar{\mu}_{1 \infty} \mu_{2 \infty}\left(\partial_{z} \mu_{1 \infty}\right) \Psi_{1, \mathrm{DS}}^{a}
\end{array}\right) .
\end{gathered}
$$

Therefore, the gauge and adjoint transformations are commutative under the condition (5.11).

Lemma 5.4. Spectral data of the adjoint problem (4.3) are related to the spectral data of the direct problem (2.4) as

$$
b^{a}(k)=\overline{b(k)} \quad k_{j}^{a}=k_{j} \quad z_{j}^{a}=z_{j} \quad c_{j}^{a}=-\bar{c}_{j} .
$$

Proof. Applying the gauge transformations of lemmas 3.1 and 5.1 to spectral representations (2.28) and (4.24) for $\boldsymbol{\mu}(z, k)$ and $\boldsymbol{\mu}^{a}(z, k)$, we obtain the spectral representations for $\boldsymbol{\mu}_{\mathrm{DS}}(z, k)$ and $\boldsymbol{\mu}_{\mathrm{DS}}^{a}(z, k)$ :

$\boldsymbol{\mu}_{\mathrm{DS}}(z, k)=\mathbf{e}_{1}+\sum_{j=1}^{n} \frac{\mathrm{i}}{k-k_{j}}\left(m_{\infty}\right)^{-1} \boldsymbol{\Phi}_{j}(z)+\frac{1}{2 \pi \mathrm{i}} \iint \frac{\mathrm{d} k^{\prime} \wedge \mathrm{d} \bar{k}^{\prime}}{k^{\prime}-k} b\left(k^{\prime}\right)\left(m_{\infty}\right)^{-1} \mathbf{N}_{\mu}\left(z, k^{\prime}\right)$

$\boldsymbol{\mu}_{\mathrm{DS}}^{a}(z, k)=\mathbf{e}_{2}-\sum_{j=1}^{n} \frac{\mathrm{i}}{k-k_{j}^{a}}\left(m_{\infty}^{a}\right)^{-1} \boldsymbol{\Phi}_{j}^{a}(z)-\frac{1}{2 \pi \mathrm{i}} \iint \frac{\mathrm{d} k^{\prime} \wedge \mathrm{d} \bar{k}^{\prime}}{k^{\prime}-k} b^{a}\left(k^{\prime}\right)\left(m_{\infty}^{a}\right)^{-1} \mathbf{N}_{\mu}^{a}\left(z, k^{\prime}\right)$.

Similarly, applying gauge transformations to the limiting formulae (2.26) and (4.23), we obtain $\lim _{k \rightarrow k_{j}}\left(\boldsymbol{\mu}_{\mathrm{DS}}(z, k)-\frac{\mathrm{i}}{k-k_{j}}\left(m_{\infty}\right)^{-1} \boldsymbol{\Phi}_{j}(z)\right)=\left(z+z_{j}\right)\left(m_{\infty}\right)^{-1} \boldsymbol{\Phi}_{j}(z)+c_{j}\left(m_{\infty}\right)^{-1} \boldsymbol{\Phi}_{j}^{\prime}(z)$ 


$$
\lim _{k \rightarrow k_{j}^{a}}\left(\boldsymbol{\mu}_{\mathrm{DS}}^{a}(z, k)+\frac{\mathrm{i}}{k-k_{j}^{a}}\left(m_{\infty}^{a}\right)^{-1} \boldsymbol{\Phi}_{j}^{a}(z)\right)=\left(z+z_{j}^{a}\right)\left(m_{\infty}^{a}\right)^{-1} \boldsymbol{\Phi}_{j}^{a}(z)+c_{j}^{a}\left(m_{\infty}^{a}\right)^{-1} \boldsymbol{\Phi}_{j}^{a \prime}(z) .
$$

Since eigenfunctions of the DS-II and IS-II equations are related by the gauge transformation as

$$
\begin{array}{ll}
\left(m_{\infty}\right)^{-1} \mathbf{N}_{\mu}=\mathbf{N}_{\mu, \mathrm{DS}} & \left(m_{\infty}^{a}\right)^{-1} \mathbf{N}_{\mu}^{a}=\mathbf{N}_{\mu, \mathrm{DS}}^{a} \\
\left(m_{\infty}\right)^{-1} \boldsymbol{\Phi}_{j}=\boldsymbol{\Phi}_{j, \mathrm{DS}} & \left(m_{\infty}^{a}\right)^{-1} \boldsymbol{\Phi}_{j}^{a}=\boldsymbol{\Phi}_{j, \mathrm{DS}}^{a} \\
\left(m_{\infty}\right)^{-1} \boldsymbol{\Phi}_{j}^{\prime}=\boldsymbol{\Phi}_{j, \mathrm{DS}}^{\prime} & \left(m_{\infty}^{a}\right)^{-1} \boldsymbol{\Phi}_{j}^{\prime a}=\boldsymbol{\Phi}_{j, \mathrm{DS}}^{\prime a}
\end{array}
$$

we find the representations (5.32) and (5.28) and limiting formulae (5.29) and (5.31) for the DS-II equations with the same spectral data $\left\{b(k), k_{j}, c_{j}, z_{j}\right\}$ and $\left\{b^{a}(k), k_{j}^{a}, c_{j}^{a}, z_{j}^{a}\right\}$. The symmetry relations (5.27) were proved for the DS-II equations in [PSO0b].

The relation (5.11) between $m_{\infty}^{a}(z)$ and $m_{\infty}(z)$ closes the system of equations (4.24) and (4.25) for eigenfunctions of the adjoint problem. For the explicit example of the single lump solution of the IS-II equations, we find from (3.22), (3.23) and (5.11) that

$$
\mu_{1 \infty}^{a}=\frac{-\mathrm{i} \overline{c_{j}} \mathrm{e}^{\mathrm{i}\left(k_{j} z+k_{j}^{-} z\right)}}{\bar{k}_{j}\left(\left|z+z_{j}\right|^{2}+\left|c_{j}\right|^{2}\right)} \quad \mu_{2 \infty}^{a}=1-\frac{\mathrm{i}\left(\bar{z}+\bar{z}_{j}\right)}{k_{j}\left(\left|z+z_{j}\right|^{2}+\left|c_{j}\right|^{2}\right)} .
$$

The algebraic system (4.25) for $b(k)=0$ and $n=1$ becomes closed with (5.11) and (5.27) and it has an exact solution:

$$
\begin{aligned}
\Phi_{1 j}^{a}(z) & =\frac{\bar{c}_{j} \mathrm{e}^{\mathrm{i}\left(k_{j} z+\overline{k_{j}} z\right)}\left[\left|z+z_{j}\right|^{2}+\left|c_{j}\right|^{2}+\frac{\mathrm{i}}{\bar{k}_{j}}\left(z+z_{j}-\bar{z}-\bar{z}_{j}\right)\right]}{\left(\left|z+z_{j}\right|^{2}+\left|c_{j}\right|^{2}\right)^{2}} \\
\Phi_{2 j}^{a}(z) & =\frac{\left(\bar{z}+\bar{z}_{j}\right)\left(\left|z+z_{j}\right|^{2}+\left|c_{j}\right|^{2}\right)-\frac{\mathrm{i}}{k_{j}}\left(\left(\bar{z}+\bar{z}_{j}\right)^{2}+\left|c_{j}\right|^{2}\right)}{\left(\left|z+z_{j}\right|^{2}+\left|c_{j}\right|^{2}\right)^{2}} .
\end{aligned}
$$

Using the gauge transformation $\boldsymbol{\mu}_{\mathrm{DS}}^{a}=\left(m_{\infty}^{a}\right)^{-1} \boldsymbol{\mu}^{a}$, we find the eigenfunction of the adjoint problem for DS-II equations from (5.27) and (5.29):

$$
\boldsymbol{\mu}_{\mathrm{DS}}^{a}(z, k)=\mathbf{e}_{2}-\frac{\mathrm{i}}{k-k_{j}} \boldsymbol{\Phi}_{j, \mathrm{DS}}^{a}(z)
$$

where the bound state of the adjoint problem $\Phi_{j, \mathrm{DS}}^{a}(z)$ is

$$
\Phi_{1 j, \mathrm{DS}}^{a}(z)=\frac{\bar{c}_{j} \mathrm{e}^{\mathrm{i}\left(k_{j} z+\overline{k_{j} z}\right)}}{\left|z+z_{j}\right|^{2}+\left|c_{j}\right|^{2}} \quad \Phi_{2 j, \mathrm{DS}}^{a}(z)=\frac{\bar{z}+\bar{z}_{j}}{\left|z+z_{j}\right|^{2}+\left|c_{j}\right|^{2}} .
$$

\section{Applications}

We use the main results of our analysis to study the spectral decomposition and surface representation theorems for the Ishimori-II equations.

The spectral decomposition theorem for the DS-II equations was proved in [PSO0b]. Structural instability of lump solutions of the DS-II equations was studied by means of perturbation series expansions over a complete set of eigenfunctions of the linear problem (3.1). Using orthogonality and completeness of eigenfunctions of the linear problem for the DS-II equations and the gauge transformation between DS-II and IS-II equations, we construct a basis of the spectral decomposition for the IS-II equations. Similar to [PS00b], the 
perturbation series expansions can be used to prove structural instability of lump solutions of the IS-II equations.

Theorem 6.1. An arbitrary scalar function $f(z) \in L^{2}\left(\mathbb{R}^{2}\right)$ can be decomposed through the set of eigenfunctions $S=\left\{\left[m_{\infty}^{-1} \mathbf{N}_{\mu}\right]_{2}(z, k),\left[m_{\infty}^{-1} \boldsymbol{\Phi}_{j}\right]_{2}, j=1, \ldots, n\right\}$ as follows

$$
f(z)=\iint \mathrm{d} k \wedge \mathrm{d} \bar{k} \alpha(k)\left[m_{\infty}^{-1} \mathbf{N}_{\mu}\right]_{2}(z, k)+\sum_{j=1}^{n} \alpha_{j}\left[m_{\infty}^{-1} \boldsymbol{\Phi}_{j}\right]_{2}(z)
$$

where

$\alpha(k)=-\frac{1}{4 \pi^{2}}\left(\left[\left(m_{\infty}^{a}\right)^{-1} \mathbf{N}_{\mu}^{a}\right]_{1}(k), f\right) \quad \alpha_{j}=\frac{1}{2 \pi \mathrm{i}}\left(\left[\left(m_{\infty}^{a}\right)^{-1} \boldsymbol{\Phi}_{j}^{a}\right]_{1}, f\right)$

and the inner product for scalar functions is defined by

$$
\left(g\left(k^{\prime}\right), f(k)\right)=\iint g\left(z, k^{\prime}\right) f(z, k) \mathrm{d} z \wedge \mathrm{d} \bar{z} .
$$

Proof. The proof follows from propositions 3.3-3.5 of [PSO0b].

Orthogonality and completeness relations for eigenfunctions of the IS-II equations can be formulated explicitly as follows.

Lemma 6.2. Eigenfunctions $\left[m_{\infty}^{-1} \mathbf{N}_{\mu}\right]_{2}(z, k)$ and $\left[m_{\infty}^{-1} \mathbf{\Phi}_{j}\right]_{2}$ are orthogonal with respect to the eigenfunctions $\left[\left(m_{\infty}^{a}\right)^{-1} \mathbf{N}_{\mu}^{a}\right]_{1}(z, k)$ and $\left[\left(m_{\infty}^{a}\right)^{-1} \boldsymbol{\Phi}_{j}^{a}\right]_{1}$ according to the orthogonality relations:

$$
\begin{aligned}
& \left(\left[\left(m_{\infty}^{a}\right)^{-1} \mathbf{N}_{\mu}^{a}\right]_{1}\left(k^{\prime}\right),\left[m_{\infty}^{-1} \mathbf{N}_{\mu}\right]_{2}(k)\right)=-2 \pi^{2} i \delta\left(k^{\prime}-k\right) \\
& \left(\left[\left(m_{\infty}^{a}\right)^{-1} \mathbf{N}_{\mu}^{a}\right]_{1}\left(k^{\prime}\right),\left[m_{\infty}^{-1} \boldsymbol{\Phi}_{j}\right]_{2}\right)=0 \\
& \left(\left[\left(m_{\infty}^{a}\right)^{-1} \boldsymbol{\Phi}_{j}^{a}\right]_{1},\left[m_{\infty}^{-1} \mathbf{N}_{\mu}\right]_{2}(k)\right)=0 \\
& \left(\left[\left(m_{\infty}^{a}\right)^{-1} \boldsymbol{\Phi}_{l}^{a}\right]_{1},\left[m_{\infty}^{-1} \boldsymbol{\Phi}_{j}\right]_{2}\right)=2 \pi i \delta_{j, l} .
\end{aligned}
$$

Corollary 6.3. The orthogonality relations for eigenfunctions of the IS-II equations take the form of weighted inner vector products defined by (4.2)

$$
\left(\left[\left(m_{\infty}^{a}\right)^{-1} \mathbf{g}^{a}\right]_{1},\left[m_{\infty}^{-1} \mathbf{f}\right]_{2}(k)\right)=\left\langle\sigma \overline{g_{1}^{a}} \mathbf{e}_{1} \mid f_{2} \mathbf{e}_{2}\right\rangle-\frac{1}{2}\left\langle\sigma \overline{\mathbf{g}^{a}} \mid\left(P-\sigma_{3}\right) \mathbf{f}\right\rangle
$$

Remark 6.4. The orthogonality relations (6.4)-(6.7) for the IS-II equations can be proved directly from the linear systems (2.4) and (4.3), with the use of weighted inner products in the right-hand side of (6.8).

Lemma 6.5. Eigenfunctions $\left[m_{\infty}^{-1} \mathbf{N}_{\mu}\right]_{2}(z, k)$ and $\left[m_{\infty}^{-1} \boldsymbol{\Phi}_{j}\right]_{2}$ are complete with respect to the adjoint eigenfunctions $\left[\left(m_{\infty}^{a}\right)^{-1} \mathbf{N}_{\mu}^{a}\right]_{1}(z, k)$ and $\left[\left(m_{\infty}^{a}\right)^{-1} \boldsymbol{\Phi}_{j}^{a}\right]_{1}$ according to the completeness relation:

$$
\begin{aligned}
\delta\left(z^{\prime}-z\right)=- & \frac{1}{2 \pi^{2} \mathrm{i}} \iint \mathrm{d} k \wedge \mathrm{d} \bar{k}\left[\left(m_{\infty}^{a}\right)^{-1} \mathbf{N}_{\mu}^{a}\right]_{1}\left(z^{\prime}, k\right)\left[m_{\infty}^{-1} \mathbf{N}_{\mu}\right]_{2}(z, k) \\
& -\frac{1}{\pi} \sum_{j=1}^{n}\left[\left(m_{\infty}^{a}\right)^{-1} \boldsymbol{\Phi}_{j}^{a}\right]_{1}\left(z^{\prime}\right)\left[m_{\infty}^{-1} \boldsymbol{\Phi}_{j}\right]_{2}(z)
\end{aligned}
$$


The linear problem for the DS-II equations and its adjoint was used in [K96, K00] to parametrize surfaces and integrable evolutions of surfaces in $\mathbb{R}^{3}$ and $\mathbb{R}^{4}$. Their results generalize the classical Weierstrass-Enneper representation for conformal parametrizations of minimal surfaces in $\mathbb{R}^{3}$, or stationary Lagrangian surfaces in $\mathbb{R}^{4}$ [HR02]. Similarly, we formulate the surface representation theorem by using the gauge transformation between the DS-II and IS-II equations.

Theorem 6.6. Define the generalized Weierstrass formulae

$$
\begin{aligned}
X^{1}+\mathrm{i} X^{2} & =\int_{\Gamma}\left(-\phi_{1} \phi_{2} \mathrm{~d} z^{\prime}+\psi_{1} \psi_{2} \mathrm{~d} \bar{z}^{\prime}\right) \\
X^{1}-\mathrm{i} X^{2} & =\int_{\Gamma}\left(\bar{\psi}_{1} \bar{\psi}_{2} \mathrm{~d} z^{\prime}-\bar{\phi}_{1} \bar{\phi}_{2} \mathrm{~d} \bar{z}^{\prime}\right) \\
X^{3}+\mathrm{i} X^{4} & =\int_{\Gamma}\left(\bar{\psi}_{2} \phi_{1} \mathrm{~d} z^{\prime}+\psi_{1} \bar{\phi}_{2} \mathrm{~d} \bar{z}^{\prime}\right) \\
X^{3}-\mathrm{i} X^{4} & =\int_{\Gamma}\left(\bar{\psi}_{1} \phi_{2} \mathrm{~d} z^{\prime}+\psi_{2} \bar{\phi}_{1} \mathrm{~d} \bar{z}^{\prime}\right)
\end{aligned}
$$

where

$\left(\phi_{1}, \psi_{1}\right)^{\mathrm{T}}=m_{\infty}^{-1} \boldsymbol{\mu}(z, k) \mathrm{e}^{\mathrm{i} k z} \quad\left(\psi_{2},-\phi_{2}\right)^{\mathrm{T}}=\left(m_{\infty}^{a}\right)^{-1} \boldsymbol{\mu}^{a}(z, k) \mathrm{e}^{-\mathrm{i} k z}$

and $\Gamma$ is a contour in $\mathbb{C}$. Then, the Weierstrass formulae define a conformal immersion of a surface into $\mathbb{R}^{4}: X^{i}(z, \bar{z}): \mathbb{C} \mapsto \mathbb{R}^{4}$. The induced metric of the surface is

$$
\mathrm{d} s^{2}=u_{1} u_{2} \mathrm{~d} z \mathrm{~d} \bar{z}
$$

where $u_{\alpha}=\left|\phi_{\alpha}\right|^{2}+\left|\psi_{\alpha}\right|^{2}, \alpha=1,2$, and the Gaussian and mean curvatures are respectively

$K=-\frac{2}{u_{1} u_{2}} \frac{\partial^{2}}{\partial z \partial \bar{z}} \log \left(u_{1} u_{2}\right) \quad H^{2}=\frac{4}{u_{1} u_{2}} \frac{\left|\bar{\mu}_{1 \infty} \partial_{\bar{z}} \bar{\mu}_{2 \infty}-\bar{\mu}_{2 \infty} \partial_{\bar{z}} \bar{\mu}_{1 \infty}\right|^{2}}{\left(\left|\mu_{1 \infty}\right|^{2}+\left|\mu_{2 \infty}\right|^{2}\right)^{2}}$.

Proof. Proof follows from theorem 3 and proposition 1 in [K00].

\section{Acknowledgments}

D Wu would like to thank people at the Department of Mathematics of McMaster University for the hospitality during her visit in the spring of 2002. The research is supported in part by NSERC grant RGP238931-01.

\section{References}

[AC91] Ablowitz M J and Clarkson P A 1991 Solitons, Nonlinear Evolution Equations and Inverse Scattering (Cambridge: Cambridge University Press)

[APP89] Arkadiev V A, Pogrebkov A K and Polyvanov M C 1989 Inverse scattering transform method and soliton solutions for Davey-Stewartson II equation Physica D 36189

[BC84] Beals R and Coifman R R 1984 Scattering and inverse scattering for the first order systems Comm. Pure Appl. Math. 37 39-90

[BC88] Beals R and Coifman R R 1988 The Spectral Problem for the Davey Stewartson and Ishimori Hierarchies: Integrability and Spectral Methods (Manchester: Manchester University Press)

[FA84] Fokas A S and Ablowitz M J 1984 On the inverse scattering transform of multidimensional nonlinear equations related to first-order systems in the plane J. Math. Phys. 25 2494-505

[GK96] Gadyl'shin G G and Kiselev O M 1996 On solitonless structure of the perturbed soliton solution for the Davey-Stewartson equation Theor. Math. Phys. 106 167-73 
[HR02] Helein F and Romon P 2002 Hamiltonian stationary Lagrangian surfaces in $\mathbb{C}^{2}$ Comm. Anal. Geom. 10 79-126

[I84] Ishimori Y 1984 Multi-vortex solutions of a two-dimensional nonlinear wave equation Prog. Theor. Phys. 72 33-7

[K93] Konopelchenko B G 1993 Solitons in Multidimensions (Singapore: World Scientific)

[K96] Konopelchenko B G 1996 Induced surfaces and their integrable dynamics, Stud. Appl. Math. 96 9-51

[K00] Konopelchenko B G 2000 Weierstrass representations for surfaces in 4D spaces and their integrable deformations via DS hierarchy Ann. Glob. Anal. Geom. 18 61-74

[LS89] Lipovskii V D and Shirokov A V 1989 Example of gauge equivalence of multidimensional integrable equations, Funct. Anal. Appl. 23 65-6

[PS00a] Pelinovsky D E and Sulem C 2000 Eigenfunctions and eigenvalues for a scalar Riemann-Hilbert problem associated to inverse scattering Comm. Math. Phys. 208 713-60

[PS00b] Pelinovsky D E and Sulem C 2000 Spectral decomposition for the Dirac system associated to the DSII equation Inverse Probl. 16 59-74

[S92] Soyeur A 1992 The Cauchy problem for the Ishimori equation J. Funct. Anal. 105 233-55 